\title{
NOTAS PARA UNA APROXIMACIÓN A LOS ESTUDIOS DE TRADUCCIÓN EN BRASIL
}

\author{
Alberto GARCÍA MARCOS \\ azherox@gmail.com \\ (Instituto Cervantes, São Paulo)
}

RESUMEN: El presente trabajo trata de aportar una aproximación al desarrollo de la traducción como disciplina académica en Brasil. Parte de una delimitación cronológica y temática, la Independencia del país, para abordar con más detalle lo que han sido, especialmente, la cuatro última décadas. En ellas se observa la progresiva incorporación de estos estudios en el panorama universitario brasileño, como resultante de una actividad traductora previa y del propio ritmo de la sociedad.

Palabras clave: Traducción, Brasil, formación superior

ABSTRACT: This paper tries to provide an approach to the development of translation as an academic discipline in Brazil. Part of a chronological and thematic delimitation, the Independence of the country, to address in more detail what they have been, especially, the last four decades. They show the progressive incorporation of these studies in the Brazilian university landscape, as a result of a previous translation activity and the pace of society itself.

Keywords: Translation, Brazil, higher education 


\section{La traducción en Latinoamérica. Intento de delimitación}

Nunca es fácil precisar con exactitud los límites de cualquier aproximación a la historia de una disciplina científica. En principio, todo ejercicio de esa naturaleza debería decidir, aunque fuera de forma implícita, tres grandes cuestiones (F. García Marcos, 2019):

A. El segmento temporal entre el que va a discurrir.

B. Su ámbito temático de competencia.

C. Su secuencia descriptiva

D. La forma expositiva en que va a ser abordado.

En el caso que me ocupa aquí, los estudios de traducción en Brasil, la última de las cuestiones anteriores no presenta demasiados inconvenientes. Se trata de realizar una primera aproximación en forma de artículo científico, lo que implica un formato ya preestablecido. A la vez, exime de mayores profundidades acerca del componente narratológico del quehacer historiográfico. No hay posibilidad de desarrollarlo y, por lo tanto, no parece necesario discutirlo. El segundo de los componentes anteriores, el ámbito temático de competencia tampoco ofrece mayores dudas. El objetivo final que se persigue aquí consiste en mostrar -y, en la medida de lo posible, tratar de explicar- cuál es la situación en la que se encuentran los estudios de traducción en Brasil. Como es natural, a ello se ha llegado en un contexto inmediato, el de la propia práctica traductora y el de la 


\section{A. García Marcos}

2019. "Notas para una aproximación a los estudios de traducción en Brasil" Sabir. International Bulletin of Applied Linguistics, 1: 67-86

actividad traductológica en este país. De manera que parece conveniente hacerse cargo del recorrido que va desde esas prácticas e inquietudes sociales hasta su concreción en formato académico. Para dar cuenta de todo ello va a seguirse una secuencia -tercer nivel anterior- de tipo básicamente cronológico. Entiendo que de esa forma queda reflejada reflejada de manera más inmediata esa trayectoria a la que acabo de hacer referencia, desde la sociedad civil a la académica.

Queda, por tanto, establecer el marco cronológico. Uno de los límites, el final, no presenta mayores problemas. Es obvio que el objeto de atención de este trabajo concluye en la etapa contemporánea. El inicio ya recomienda mayores precauciones. Existe una corriente reciente que vincula la traducción con los procesos de colonización en América. Bueno y Vega (2011), o el propio Vega (2012) en solitario, resaltan ese rol evangélico de la traducción, sobre todo entre los franciscanos. Bastin (2017) le asigna un papel determinante en la misma configuración de la identidad latinoamericana.

Sin embargo, no parece ser esa la única lectura posible de ese episodio histórico. F. García Marcos (2008) destaca otro factor, solo en parte complementario, cuando aborda las causas que llevan a la aparición y desarrollo de la lingüística misionera. Los jesuítas comprenden que no se puede realizar una evangelización en lenguas europeas, por lo que deciden abandonar la prédica en latín o en español. Lo imperioso, desde el punto de vista religioso, era evangelizar a los indígenas para poder bautizarlos con fundamentos $y$, en consecuencia, permitirles alcanzar la salvación. Eso se traduce en una extraordinaria actividad normativizadora de las lenguas amerindias, responsable en gran medida de la supervivencia de muchas de ellas hasta nuestros días. Por otra parte, el propio F. García Marcos (2012) señala que la traducción y la interpretación entre el español y las lenguas nativas fue más allá de lo estrictamente religioso. Formó parte, en gran medida, del modelo colonizador. Al menos en los virreinatos españoles 


\section{A. García Marcos}

2019. "Notas para una aproximación a los estudios de traducción en Brasil" Sabir. International Bulletin of Applied Linguistics, 1: 67-86

había un bilingüismo sensiblemente extendido en algunas capas sociales $y$, por lo demás, estaba institucionalizada la figura del intérprete en la administración de justicia.

A la vista de lo anterior, parece prudente adoptar la postura de Pagny (2014: 208), quien toma en cuenta todos esos antecedentes, pero tampoco termina de adoptarlos con claridad como un precedente inequívoco de traducción latinoamericana propiamente dicho. Sin duda aportan huellas de la traducción en la geografía americana. Pero ya resulta más cuestionable considerarla traducción de esas sociedades. Por consiguiente, aquí me centraré única y exclusivamente en Brasil y en la sociedad brasileña, después de la Independencia, con independencia del sustrato, mayor o menor, que pueda haber de actividad traductora.

\section{Los inicios de la actividad traductora en Brasil}

En un trabajo relativamente reciente, Milton (2014: 3), uno de los grandes referentes traductológicos contemporáneos en Brasil no vacila en situar con exactitud el inicio de los estudios de traducción en el país, sin duda bien avanzado el Siglo XX. Entiene que edse honor, y esa responsabilidad, cabe atribuírsela a Paulo Rónai (1907-1992), quien entre 1945 y 1955 se encarga de traducir y publicar la Comédie Humaine de Balzac. Rónai era un inmigrante húngaro que en ese segmento temporal consigue dar a la luz las 17 entregas de la que consta la obra de Balzac. Rónai ejerció de coordinador de un equipo de traducción integrado por 14 profesionales, dentro de un proyecto de Globo, casa editora radicada en Porto Alegre. 


\section{A. García Marcos}

2019. "Notas para una aproximación a los estudios de traducción en Brasil" Sabir. International Bulletin of Applied Linguistics, 1: 67-86

Ese primer proyecto tuvo continuidad en Mar de Histórias. En esta ocasión aparecieron diez volúmenes. En ellos se recogían cuentos procedentes de distintos lugares del mundo. Se trataba, pues, de un proyecto más ambicioso en cuanto al número de lenguas que realmente abría un horizonte traductor más amplio y genérico.

Tradujo también del portugués a otras lenguas. En concreto, realizó la versión francesa de Memórias de um sargento de milícias (1944), de Manuel Antonio de Almeida y la húngara de Mensagem do Brasil: poetas brasileiros contemporâneos (1939) que, en sentido estricto, es su primera producción como traductor.

El propio Rónai realiza también contribuciones teóricas, como es lógico, estrechamente ligadas a su experiencia como traductor y editor de traducciones. En plena época la edición brasileña de Balzac, publica Escola de Tradutores (1952). Casi tres décadas después aparece $A$ Tradução Vivida (1981), una obra que en gran medida hace balance de la experiencia de los años anteriores. No es, en todo caso, su única producción científica. Tuvo también apariciones en gramática de lenguas extranjeras, enseñanza de lenguas o cultura clásica. A esa parte de su producción pertenecen Gramática completa do francês (1969), Como aprendi o português e outras aventuras (1956), Não perca o seu latim (1980), Dicionário francês-português (1980), Gradus primus (1943), Gradus secundus (1986).

Rónai, además, encarna en gran medida el prototipo de traductor que va a predominar en Brasil durante esa primera etapa de arranque de la actividad traductora. Intelectuales en sentido amplio, profesionales de distinta procedencia, políglotas, acceden a la traducción como una parte de sus inquietudes culturales, también desde el convencimiento de que mediante ella se contribuye a la difusión de ideas y a la aproximación de los grandes hitos de la literatura al público brasileño. Las siguientes referencias que menciona Milton en ese mismo lugar 


\section{A. García Marcos}

2019. "Notas para una aproximación a los estudios de traducción en Brasil" Sabir. International Bulletin of Applied Linguistics, 1: 67-86

son los hermanos Campos, Haroldo y Augusto. Ambos abogados de profesión son traductores prolíficos, que terminan realizando aportaciones teóricas, como en el caso de Rónai, aunque nunca llegaron a ejercer como docentes en traducción o en traductología. Sobre todo, activos en las décadas de los 80 y 90 , a Haroldo se le deben las versiones brasileñas de Joyce, Goethe, Khlebhikov, Maiokovski, Homero, Mallarmé o, entre otros, Pound. Se le reconoció un solvente dominio de lenguas extranjeras -latín, inglés, español y francés- que indudablemente utilizó en sus traducciones. Fue una figura literaria relevante, siendo uno de los fundadores del Movimiento Concretista en 1956, grupo de poetas con gran peso en la sociedad literaria brasileña. Augusto aportó una nómina en parte similar, con trabajos sobre Mallarmé, Joyce, Pound, Maiokovski, Donne, o Cummings. Augusto también ha sido poeta, co-partícipe de la fundación del movimiento concretista, aunque ha evolucionado hacia formas mucho más vanguardistas. Es uno de los referentes de la poesía virtual brasileña. De su colaboración con el artista visual Moysés Baumstein surgió la elaboración de poemas holográficas, todo un símbolo de los nuevos caminos de la poesía que abandona el papel. Al margen de las coincidencias en preferencias literarias, lo cierto es que entre ambos conforman listado de autores muy respetable, incluyendo referencias de cabecera en la historia de la literatura, en varios períodos además. La vertiente teórica de Haroldo surge en $A$ Arte no Horizonte do Provável(1969), A Operação do Texto (1976) y Metalinguagem e Outras Metas (1992). Augusto tampoco desdeñó ese segundo tipo de publicaciones, ámbito teórico del que se ocupó Verso, Reverso, Controverso (1976) y O Anticrítico (1986).

La contribución teórica de los hermanos Campos, muy vinculada a su propia actividad como traductores, está centrada en el dominio literario. Más en concreto, se centra en sus componentes sonoros y visuales de la traducción. Haroldo formula la noción "transcriação", mediante la que trata de superar la 


\section{A. García Marcos}

2019. "Notas para una aproximación a los estudios de traducción en Brasil" Sabir. International Bulletin of Applied Linguistics, 1: 67-86

dualidad forma/contenido. A cambio propone concentrarse en la forma poética y en la estructura fono-semántica, tratando de conjugar y acompasar el trabajo sobre el significado y sobre la forma. No se trata, por tanto, de apostar por una traducción libre, sino más bien de recrear un proceso de creación literaria en otra lengua. En ese objetivo entienden que el traductor no debe de tener cortapisas, incluso creando neologismo si fuera necesario.

Haroldo y Augusto Campos tienen una importancia crucial para el desarrollo de la traducción en Brasil, no solo por su actividad como traductores y por sus escritos teóricos, sino porque instituyen un modelo que será seguido por otros autores. José Paulo Paes (1926-1998), químico de formación y profesión, poeta como los hermanos Campos, se ocupó de Charles Dickens, Joseph Conrad, Konstantinos Kavafis, Paul Eluard, Hölderlin, Rilke, o, entre otros, Ovidio y Nikos Kazantzakis. Como los autores anteriores, cuenta con su producción teórica, reunida en Tradução: a Ponte Necessária (1990). Paes sigue en líneas generales los planteamientos de Haroldo y Augusto Campos, aunque disiente en relación con la oportunidad de crear neologismos.

La siguiente figura de ese arranque de los estudios de traducción en Brasil es Nelson Ascher (1958). Médico de formación académica, sus traducciones están más concentradas en el ruso (Pushkin), como en la poesía húngara. Se pone de manifiesto nuevamente una gran riqueza políglota, habida cuenta de que el húngaro es una lengua no-indeuropea, alejada en consecuencia tipológicamente tanto del ruso como del portugués, si bien es la lengua materna de su familia, emigrada desde Hungría a Brasil. Como teórico, Ascher inicialmente siguió las directrices marcadas por Haroldo de Campos, aunque en sus trabajos más recientes se muestra más abierto a nuevas corrientes traductológicas (Milton, 2014: 6). Asimismo, cabe reseñar que es poeta en activo. De él se ha destacado su capacidad para aunar recursos procedentes de la tradición clásicas con formas 


\section{A. García Marcos}

2019. "Notas para una aproximación a los estudios de traducción en Brasil" Sabir. International Bulletin of Applied Linguistics, 1: 67-86

más vanguardistas de la poesía contemporánea. Por lo demás, como recuerda uno de los referentes contemporáneos de la traductología brasileña, Walter Carlos Costa, en una entrevista reciente (Lazaro y Costa: 2014), la propia configuración sociológica del país inclinaba hacia dominios vinculados con la traducción. El propio Costa, natural del estado de Sao Paulo, reconoce haberse inclinado a ella en gran medida por su experiencia directa con el multilingüismo con el que convivía a diario, en una zona con fuertes contingentes migratorios, procedentes de otros lugares de Brasil y de fuera del país.

\section{La consolidación a partir de la segunda mitad de los $\mathbf{8 0}$}

A finales de los 80 se produce un cambio sociológico muy sustancial para el futuro de los estudios de traducción en Brasil. Hasta ese momento la traducción había ido emergiendo junto a las inquietudes de literatos y hombres de la cultura, procedentes de campos profesionales muy diversos, que compartían el común denominador de su ostensible conocimiento de lenguas y su espíritu cosmopolita. A partir de ese momento la universidad se encargará de encauzar esas preocupaciones y esas líneas de trabajo. Lo hará, además, de una forma constructiva, aprovechando y dando valor al trabajo acumulado hasta ese momento. Los traductores que acaban de mencionarse pasarán a tener presencia en las aulas universitarias. Es cierto que no se integran por completo y de inmediato a la enseñanza superior. Pero no menos verdad es que su mera presencia en las aulas de algunas universidades brasileñas supuso abrir un campo de preocupaciones, el traductológico, que terminaría asentándose en el entramado universitario brasileño. Si Folha de São Paulo, Folhetim (1977-1989) había sido el gran difusor de las preocupaciones traductológicas de los autores anteriores entre la sociedad culta, el testigo lo toma Tradução e Comunicação 


\section{A. García Marcos}

2019. "Notas para una aproximación a los estudios de traducción en Brasil" Sabir. International Bulletin of Applied Linguistics, 1: 67-86

(1981-1986), ya dentro de una estructura universitaria, la de la Faculdade Ibero americana (después Universidade Ibero - americana UNIBERO).

Con los protagonistas de ese primer momento de arranque de la traductología en Brasil sucederá otro tanto. Haroldo Campos participa en la PUC dentro de un máster sobre comunicación y semiótica. Más tarde hace lo propio en la UNICAMP, en el Instituto de Estudos Avançados en la USP y en el Departamento de Teoría de la Literatura de la Universidad de São Paulo, poco antes de su muerte. La traducción empieza a encontrar hueco en la sociedad académica a través de áreas de conocimiento próximas, de campos de interés científicos no alejados de los que serían sustantivamente propios, siguiendo por lo demás un procedimiento bastante estandarizado para esta clase de situaciones.

Al hilo de esa actividad, siguiendo pautas análogas, R. Arrojo y P. Ottoni trabajan en el Departamento de Lingüística Aplicada en el Instituto de Estudos da Linguagem, dentro de la UNICAMP, siguiendo una orientación deconstructivista, muy ligada a Derrida y, por tanto, dentro de la teoría de la literatura.

Es también la etapa de arranque de los primeros trabajos de tercer ciclo que tocan la cuestión de la traducción en la USP, maestrías e incluso tesis doctorales. De nuevo, las investigaciones sobre traducción aparecen vinculadas a los departamentos de letras modernas o clásicas, donde estaba radicada la enseñanza de los idiomas extranjeros, así como el de Lingüística. No sucede otro tanto con el de teoría de la literatura. Milton (2014: 7) lo atribuye a la influencia directa de Antonio Candido, el referente de la disciplina en aquella época en Brasil, quien al parecer nunca se mostró proclive a interesarse por la traducción.

Casualidad o no, empeño personal, convicción académica o cualquier otra circunstancia, el hecho es que los estudios de traducción en Brasil se encaminaron en una dirección similar a la adoptada en el panorama internacional. Por supuesto 


\section{A. García Marcos}

2019. "Notas para una aproximación a los estudios de traducción en Brasil" Sabir. International Bulletin of Applied Linguistics, 1: 67-86

que la traducción ha crecido tradicionalmente junto a la literatura y que las historias de la traducción literaria han explicado, al menos en parte, las propias historias de la literatura. Conocer qué se traducía permitía hacerse cargo de cuáles eran las influencias directas recibidas durante una determinada etapa de la historia literaria. Pero no menos verdad es que la traducción, y la interpretación como correlato oral de la misma, tenían un radio de acción considerablemente más amplio. En la antigua Mesopotamia lo primero que se documenta es la presencia de intérpretes, no de traductores literarios de otras lenguas. Están al servicio de los monarcas y realizan fundamentalmente tareas diplomáticas para parlamentar con otros estados-ciudades (F. García Marcos, 2008: 15). Otro tanto puede decirse de la traducción de textos técnicos, de escritos filosóficos, de saberes, incluso de los principales documentos religiosos de cada confesión, que han viajado de unas culturas a otras gracias a las correspondientes traducciones realizadas en cada época. Hasta época relativamente reciente, se había dado por sentado una Escuela de Traductores de Toledo, configurada a partir del siglo XIII. Quizá fuera más preciso pensar en una gran actividad traductora, con varios centros en la España cristiana durante la Edad Media (Palencia, Salamanca, Murcia), que finalmente convergió, o cristalizó, en un proyecto integrado en Toledo. Santoyo (2099) niega esa última posibilidad y prefiere limitarse a enfatizar el trabajo traductor generalizado en la España de la época. Lo cierto es que, con independencia de su alcance formal en última instancia, sí parece probado que esa actividad se propagó a partir de centros encargados de velar por las lenguas distintas del español, siempre conforme a la singularidad propia de la época (Vélez, 2017). En la otra parte de la Península, en la España musulmana, la situación no era muy distinta Gracias a esa amplitud de intereses se tradujeron al árabe -y se conservaron para el resto de la cultura humana- los textos de los grandes filósofos de la Grecia Clásica. Por lo tanto, había, vida traductora mucho más allá de la literatura. Aunque en Brasil había nacido apegada a los poetas, la 


\section{A. García Marcos}

2019. "Notas para una aproximación a los estudios de traducción en Brasil" Sabir. International Bulletin of Applied Linguistics, 1: 67-86

opción de Candido -caprichosa o no, eso queda para sus biógrafos- tampoco quedaba tan lejana de lo que habían sido otras pautas adoptadas en el panorama internacional.

La consecuencia inmediata de ello fue que en Brasil se empiezan a desarrollar a partir de aquella época estudios universitarios de traducción, inicialmente incorporados a los programas de postgrado, siempre vinculados a la lingüística, en cualquiera de sus versiones. Milton (2014: 7-8) da un listado tan exhaustivo como ilustrativo de cuanto se comenta, que sintetizo a continuación:

Tabla 1.

Estudios de postgrados en traducción en Brasil, vinculados a otros programas universitarios

\author{
Universidad \\ Universidade Católica de Río de Janeiro (PUC -RJ), \\ Universidade do Estado de Ceará (UECE) \\ Universidade Federal de Minas Gerais (UFMG \\ Universidade Federal de Paraná (UFPR), \\ Universidade Federal de Rio Grande do Sul (UFRGS) \\ Universidade Federal de Río de Janeiro (UFRJ) \\ Universidade Federal de Río de Janeiro (UFRJ) \\ Universidade do Estado de São Paulo (São José do Rio \\ Preto \\ Universidade Campinas (UNICAMP)
}

Programa

Língua e Literatura

Lingüística Aplicada

Lingüística

Língua e Literatura

Língua e Literatura

Idiomas Neolatinos

Interdisciplinario de Lingüística

Aplicada

Lingüística

Lingüística Aplicada 


\title{
A. García Marcos
}

2019. "Notas para una aproximación a los estudios de traducción en Brasil" Sabir. International Bulletin of Applied Linguistics, 1: 67-86

A ello cabe agregar otros tres centros universitarios en los que se imparten enseñanzas superiores específicas en traducción:

\section{Tabla 2}

Centros universitarios brasileños con enseñanzas específicas en traducción

\author{
Universidade Federal de Santa Catarina (UFSC) \\ Universidade de Brasilia (UNB) \\ Universidad de São Paulo (USP)
}

Esa oferta tuvo una traducción efectiva en el número de estudiantes que se incorporaron a los estudios de traducción a partir de la década de los 90. Las cifras que aportan Milton (2014: 8) son muy indicativas En ellas se pone de manifiesto que se ha experimentado una consolidación estimable a partir de la última década del pasado siglo, lo que augura un futuro estable para la traducción y la traductología dentro del ámbito universitario brasileño. A continuación, Milton (2014: 9) aporta unos datos también muy relevantes. Ese impulso se concentra de manera muy significativa en algunos centros universitarios $y$, por consiguiente, en algunas zonas de Brasil. En efecto, siempre siguiendo a Milton, es posible establecer un ranking de producción de trabajos de maestría y doctorado en traducción, que en el caso de las universidades brasileñas quedaría como sigue: 


\section{A. García Marcos}

2019. "Notas para una aproximación a los estudios de traducción en Brasil" Sabir. International Bulletin of Applied Linguistics, 1: 67-86

\section{Tabla 3.}

Rankin de universidades brasileñas según su producción de trabajos de traducción dentro de la formación de III Ciclo

$\begin{array}{ll}\text { Posición } & \text { Universidad } \\ 1 & \text { UFMG } \\ 2 & \text { UFSC } \\ 3 & \text { USP } \\ 4 & \text { UNICAMP } \\ 5 & \text { UNESP-Río Preto } \\ 6 & \text { PUC-SP } \\ 7 & \text { UFRJ }\end{array}$

Como se puede apreciar de inmediato, el interés académico por la traducción está asociado a los grandes centros sociológicos y económicos de Brasil, así como a instituciones universitarias especialmente vinculadas a las corrientes más actuales de la lingüística, y en especial de la lingüística aplicada.

Todo ello ha contado con la correspondiente vertiente complementaria en la vida académica aportada por las asociaciones especializadas y las revistas sobre traducción que también, grosso modo, surgen en torno a ese período.

\section{La situación actual}

En 1986 aparece el Grupo de Trabalho de Tradução (GT), integrado dentro de la ANPOLL (Associação Nacional de Estudos de Pos-graduação e Pesquisa em Literatura e Linguística). Por ese grupo, muy activo en la promoción de reuniones 


\section{A. García Marcos}

2019. "Notas para una aproximación a los estudios de traducción en Brasil" Sabir. International Bulletin of Applied Linguistics, 1: 67-86

bianuales, han pasado especialistas de gran peso en Brasil como Rosemary Arrojo, Cristina Carneiro, Maria Lucia Vasconcellos, Walter Costa, Marie Hélène Torres, Maria Paula Frot, Adriana Pagan o Fabio Alves. Seis años después, en la Universidad de Campinas surge ABRAPT (Associação Brasileira de Pesquisadores en Tradução), otro de los núcleos más activos en la traductología brasileña, con un curioso periplo formal. El ABRAPT surgió de Campinas, pero se radicó en Sao Paulo en primera instancia. Pero continuó con su periplo por la Universidad Federal de Minas Gerais (UFMG), la Universidad Federal de Santa Catarina (UFSC) para terminar, al menos de momento, en la Universidade Federal de Uberlândia (UFU). Eso no le ha impedido mostrarse muy activa, además de acoger en su grupo a otro buen número de reconocidos especialistas brasileños como Mario Laranjeira João Azenha, Francis Aubert, o el propio John Milton.

Entre las actividades propias, tanto de las asociaciones como de las universidades, destaca la organización de numerosos encuentros y congresos, tanto nacionales como internacionales. Milton (2014: 10) recoge una veintena de actividades en esa dirección, en las que destaca el protagonismo que tiene la ciudad de Sao Paulo, donde se han acogido una parte muy significativa de ellas. Es más, el propio Estado de Sao Paulo apoya explícitamente la realización de cursos de traducción, que cuenta con un auténtico referente de los mismos en Transfusão, actividad inspirada en Haroldo Campos, que se realiza de manera regular a partir del año 2011.

Del mismo modo, se implantan progresivamente una serie de revistas especializadas, que vienen a completar la solidificación de la implantación académica de la traductología en Brasil. Tradução e Comunicação, de UNIBERO, arranca en 1981, aunque tuvo una vida relativamente efímera, pues desapareció cinco años después, aunque fue reeditada en 2006. TradTerm (USP) aparecería en la década siguiente, en 1995, y en la actualidad cuenta con 21 números. Esa fue 


\section{A. García Marcos}

2019. "Notas para una aproximación a los estudios de traducción en Brasil" Sabir. International Bulletin of Applied Linguistics, 1: 67-86

una época muy activa, pues al año siguiente, en 1996, ve la luz Cadernos de Tradução (UFSC) que ha completado ya 26 entregas. También otro años después, ahora en 1997, Cadernos de Literatura em Tradução se suma a ese listado, completando 13 números. Tradução em Revista (PUC - RJ) tarda más en aparecer e incorpora novedades sustanciales. El primer número es de 2004, completando tres ediciones impresas. A partir de ese momento publica en línea, tendencia que han seguido las revistas más recientes como Belas Infiéis, (PosTrad/UnB) (2012- ); In-Traduções, (PGET/UFSC) (2009- ); PROFT em Revista (2011); Scientia Traductionis, (PGET/UFSC) (2005- ); Traduzires, (UnB) (2012- ) y Translatio, (UFRGS; 2011- ).

A grandes rasgos ese es el panorama que ha llegado hasta la actualidad, lo que no quiere decir que ni la traducción ni la traductología brasileñas no tenga pendientes retos de cara al futuro más o menos inmediato. Milton (2014) de nuevo, señala grandes retos

1. Terminar de mejorar el acceso a las publicaciones especializadas de fuera de Brasil, si bien Internet ha supuesto una mejora muy ostensible en las condiciones de trabajo.

2. Aumentar la producción de los teóricos de la traducción fuera de Brasil, hasta este momento poco proclives a proyectarse fuera de Brasil.

3. Terminar de consolidar la traducción como un área científica propia y autónoma, en consonancia con lo que son las principales corrientes en el panorama bibliográfico internacional.

4. Ensamblar enseñanzas superiores previas al III Ciclo, para introducir la traducción en los programas de grado universitarios, siguiendo de nuevo tendencias también presentes en el panorama académico internacional. 


\section{A. García Marcos}

2019. "Notas para una aproximación a los estudios de traducción en Brasil" Sabir. International Bulletin of Applied Linguistics, 1: 67-86

Buena parte de esos objetivos se están empezando a conseguir de manea fehaciente, lo que supone también una considerable diversificación temática en y sobre Brasil, incorporando nuevas problemáticas al panorama de la traductología brasileña. Rodríguez (2015) pasaba revista a la traducción colaborativa, comparando Brasil y Canadá. Presagiaba que se trataba de una opción llamada a tener una inmediata evolución. Brandini (2018), por su parte, examinó la recepción de Barthes en Brasil, uno de los referentes indiscutibles de la semiótica internacional, a partir de las traducciones realizadas de su obra. Caurel (2019) se ha ocupado de la traducción jurada, contrastando Brasil, Portugal y España. La traducción literaria, naturalmente, tampoco ha sido desatendida (Costa, 2012, 2013; Costa, Guerini y Torres, 2012), tanto prestando atención a las contribuciones de traductores concretos, como a su caracterización como producción lingüística idiolectal. Lesmes (2017, en fin,) introduce una problemática muy actual, como es el doblaje cinematográfico. Tampoco han faltado las obras teóricas más propiamente traductológicas (Costa y Guerini, 2012).

En una revisión panorámica de la situación de la traducción en el Brasil contemporáneo, Costa (Lazaro y Costa, 2014: 228) subrayaba que es posible caracterizarla desde el punto de vista de "la convivencia de distintos enfoques y el desarrollo de distintas interfaces de la disciplina". En ese mismo párrafo insiste en que su conexión con las principales tendencias desarrolladas en el panorama internacional resulta evidente e indiscutible. En ese aspecto reconoce una deuda respecto de la escuela funcionalista alemana. Pero, al mismo tiempo, señala que existen

\footnotetext{
"desarrollos autónomos en la investigación de la traducción literaria (entre otros, traducción de poesía, traducción comentada de textos literarios),
} 


\section{A. García Marcos}

2019. "Notas para una aproximación a los estudios de traducción en Brasil" Sabir. International Bulletin of Applied Linguistics, 1: 67-86

historia de la traducción, traducción de lengua de señas, traducción y lingüística de corpus, traducción y filosofía, traducción y antropología, traducción de textos religiosos, traducción y crítica genética, traducción y psicoanálisis".

(Lazaro y Costa, 2014: 228)

De entre ese amplio elenco, en su opinión destacarían la "traducción comentada, traducción de lengua de señas, traducción y antropología, y traducción y psicoanálisis, sectores especialmente fuertes en la PGET y, me parece, poco desarrollados en la escena internacional". Eso no excluye que considere que todavía queda mucho que hacer.

Parece, pues, que la traducción brasileña ha seguido un camino progresivo y firme. Relativamente reciente, por la propia juventud del país y de sus estructuras académicas, ha sabido en todo caso cimentar con solvencia el desarrollo de una actividad traductora, trasladarla en una transición equilibrada al dominio académico, dotándola de todos los ingredientes del mismo. A la vista de todo ello, solo cabe esperar una evolución satisfactoria y provechosa, más que en el futuro inmediato, en este mismo presente.

\section{Bibliografía}

Bastin, G.

2017. "La traducción y la conformación de la identidad latinoamericana" en Trans. Revista de traductología, 12: 11-14. 


\section{A. García Marcos}

2019. "Notas para una aproximación a los estudios de traducción en Brasil" Sabir. International Bulletin of Applied Linguistics, 1: 67-86

Brandini, L. T. (2018). "Roland Barthes en Brasil a través de las traducciones" en Transfer, 13 (1-2): 77-93.

Bueno, A. y M. A. Vega (eds.)

2011. Lingua, cultura e discorso nella traduzione dei francescani. Perugia: Università per Stranieri di Perugia.

Caurel Santiago, J. A. (2019). La traducción jurada de documentos de constancia del estado civil en Brasil, España y Portugal. Granada: Universidad de Granada, Tesis Doctoral.

Costa, W. C.

2012. "Traducción literaria, variedad e idiolecto" in Aletria, 22,1: 83-89.

2013. "Jorge Luis Borges" en F. Lafarga y L. Pegenaute (dirs.) Diccionario histórico de la traducción en Hispanoamérica. Frankfurt: Vervuert.

- y A. Guerini.

2007. Introdução aos Estudos da Tradução. Florianópolis: LANTEC/UFSC, 2007. , Guerin, A. y M. H. Torres.

2008. Literatura nacional e literatura traduzida. Rio de Janeiro: 7Letras.

García Marcos, F.

2008. Historia social de la lingüística. Barcelona: Octaedro.

2012. "Sobre los límites del concepto de conflicto lingüístico. a propósito de Colombia a partir de la independencia" en Revista de Filología, 30: 63-82.

2019. Perspectivas en historiografía lingüistica. En prensa

Lazaro, R., y W. Costa. 


\section{A. García Marcos}

2019. "Notas para una aproximación a los estudios de traducción en Brasil" Sabir. International Bulletin of Applied Linguistics, 1: 67-86

2014. "Los estudios de la traducción en Brasil: entrevista al Profesor Walter Carlos Costa" en Mutatis Mutandis, 7 (1): 223-231.

Lázaro, R.

2012. "Haroldo de Campos: recorrido por sus textos teóricos sobre traducción y estado de traducción al castellano" en Mutatis Mutandis, 5 (2): 370-390.

Lesmes, C. F. R.

2016. La traducción de canción en doblaje: un estudio descriptivo-exploratorio para español latinoamericano y portugués brasileño. São Paulo: USP.

Milton, J. (ed.)

2001: Emerging Views of Translation History in Brazil (número especial). Sao Paulo: Humanitas FFLCH/USP.

2014. "Los estudios de la traducción en Brasil" en Mutatis Mutandis, 7 (1), 3-15.

Pagni, A. 2014. "Hacia una historia de la traducción en América Latina" en Iberoamericana), 14 (56): 205-224

Rodovalho, $\mathrm{O}$.

2012. "O sonho da razão, de Nelson Ascher" en Revista Sibila. Novos e críticos. (Consulta: http://sibila.com.br/novos-e-criticos/o-sonho-da-razao-denelson-ascher/5270

Rodríguez, R. E. C.

2015. "La traducción colaborativa activista contemporánea en Canadá y en Brasil: comunidades de traducción 1.0 y 2.0 en evolución" en Tradução em Revista, 18(1), 160.

Santoyo, J. C. 
2019. "Notas para una aproximación a los estudios de traducción en Brasil" Sabir. International Bulletin of Applied Linguistics, 1: 67-86

2009. La traducción medieval en la Península Ibérica, Siglos III-XV. León: Universidad de León.

Vega, M. A.

2012. "La traducción en la obra evangelizadora y civilizatoria tras la conquista y durante la colonia en la américa hispana" en Lafarga F. y L. Pegenaute (eds.) Lengua, cultura y política en la historia de la traducción en Hispanoamérica. Vigo: Academia del Hispanismo, 263-275.

Vélez León, P.

2017. "Sobre la noción, significado e importancia de la Escuela de Toledo" en Disputatio. Philosophical Research Bulletin 6, 7: 537-579. 